Aus aktuellem Anlass

Peter Zweifel*

\title{
Die Corona-Krise: Eine politökonomische Betrachtung
}

https://doi.org/10.1515/pwp-2020-0041

Zusammenfassung: Zur Überraschung vieler Beobachter haben im Zuge der Corona-Krise sonst freiheitsliebende Bürger die zur Eindämmung der Pandemie verhängten Einschränkungen in der Ausübung ihrer Rechte sowie die massive Ausweitung des öffentlichen Sektors hingenommen oder sogar begrüßt. Umgekehrt wurden Regierungen, die sich passiv verhielten, scharf kritisiert. Peter Zweifel hat sich in diesem Beitrag das Ziel gesetzt, den Wandel in der Interaktion zwischen den Bürgern und ihrer Regierung in Folge einer neuen Gesundheitsbedrohung zu modellieren. Es zeigt sich, dass ein entscheidender Einfluss von der Zunahme der Zahlungsbereitschaft für eine Risikosenkung ausgeht.

JEL-Klassifikation: D11, D62, I12, I13, I18

Schlüsselwörter: Corona-Krise, Covid-19, Edgeworth-Box, Trade-off zwischen Gütern

\section{Ein kurzer historischer Rückblick}

Im Jahre 1347 erreichte die Pest die Halbinsel Krim, sehr wahrscheinlich auf der Seidenstraße aus China kommend (Baras und Greub 2014). Drei Jahre später erließ die Regierung von Dubrovnik das erste Quarantänegesetz; die Isolation während 40 Tagen stützte sich auf keine medizinische Erkenntnis, sondern war religiös motiviert (so berichtet die Bibel, dass Jesus während 40 Tagen in der Wüste ausharrte). Wenig später errichtete Venedig auf der Insel Lazaretto ein Pestkrankenhaus, woraus das Wort Lazarett entstand.

In der jüngeren Geschichte gilt die Spanische Grippe als die schlimmste Seuche. Sie kam aus einer amerikanischen Militärbasis, wo sie geheim gehalten wurde; doch Spanien kannte als neutrales Land im Ersten Weltkrieg keine Zensur (Farrell 2020). In drei Wellen, von denen die zweite die tödlichste war, raffte sie 3 bis 5 Prozent der damaligen Weltbevölkerung dahin. Die politische Reaktion war die gleiche wie zuvor: Quarantäne, Isolierung. Dazu kam neu auch die Schließung von Schulen (Tribelhorn 2018).

Die Parallelen mit der Corona-Krise sind offensichtlich. Das Virus kam 2019 aus China in die Hochburgen der Textil- und Bekleidungsindustrie Norditaliens. Chinesen machen in der Lombardei nicht weniger als 5,8 Prozent der Bevölkerung aus, die meisten von ihnen sind vermutlich Arbeiterinnen aus armen Provinzen Chinas, zum Beispiel aus Hubei mit der Hauptstadt Wuhan. Und wieder war die (moderne) Seidenstraße mit im Spiel, besser bekannt als „Belt and Road Initiative“, an der auch Italien teilnimmt. Von daher war es wenig erstaunlich, dass die italienische Regierung dem Rat und Vorbild aus Peking folgte und mit durchgreifenden Isolationsmaßnahmen die Wirtschaft zum Stillstand brachte. Sämtliche EU-Länder (mit ihnen auch die Schweiz) mit Ausnahme Schwedens folgten dem italienischen Beispiel.

Vor diesem Hintergrund gilt es zu erklären, weshalb es beinahe 700 Jahre nach der Pest und 100 Jahre nach der Spanischen Grippe einmal mehr zu einer massiven Ausweitung der staatlichen Kompetenzen zu Lasten der bürgerlichen Freiheit und Verfügungsgewalt über das Einkommen kommen konnte. Hatte das Wissen über Epidemien in der Zwischenzeit nicht große Fortschritte gemacht, so dass man Risiken dieser Art mit weniger einschneidenden Maßnahmen hätte begegnen können? War nicht auch die Grippewelle 2017/18 mit mehr als 25.000 Todesfällen (Deutsches Ärzteblatt 2019) ohne derart massive Interventionen vorbeigegangen?

*Kontaktperson: Peter Zweifel, Universität Zürich (Emeritus), Wulfensiedlung 24, A-9530 Bad Bleiberg,

E-Mail: peter.zweifel@uzh.ch 


\section{Die Interaktion zwischen den Bürgern und ihrer Regierung}

\subsection{Vor der Corona-Krise}

Für die Analyse der Interaktion zwischen zwei Akteuren eignet sich die bekannte Edgeworth-Box. In der Abbildung 1 werden die Güter „Verfügungsgewalt über das BIP“ und „Beherrschung der Risiken“ unterschieden. Diese Wahl lässt sich leicht begründen: Für die Bürger ist ein höheres Maß an Verfügungsgewalt über das Volkseinkommen gleichbedeutend mit einem höheren verfügbaren Einkommen und damit Konsummöglichkeiten, während aus der Sicht der Politiker ein Mehr an Verfügungsgewalt über das Bruttoinlandsprodukt mit größeren Steuereinnahmen und damit Kompetenzen und letztlich Macht einhergeht. Die Beherrschung der Risiken ist beispielsweise für die deutschen Bürger ebenfalls ein wichtiges Gut; schließlich wenden sie nur schon für die Lebensversicherung freiwillig Einmalbeiträge in der Gesamtsumme von 27,8 Milliarden Euro auf (Gesamtverband der Deutschen Versicherungswirtschaft 2019). Für die Politiker hat sie einen geringeren Stellenwert, denn sie sind gut abgeschirmt; die Zeit vor ihrem Wiederwahltermin bildet eine Ausnahme, da sie für ein fehlerhaftes Risikomanagement verantwortlich gemacht werden können.

Diese Überlegungen begründen die Lage des Punkts $E$ in der Abbildung 1. Er entspricht der Aufteilung der beiden
Güter zwischen den Bürgern und den Politikern vor dem Eintreten der Corona-Krise. Einfachheitshalber (wenn auch nicht notwendigerweise realistisch angesichts der Unvollkommenheit des Marktes für politischen Einfluss) bildet er ein Pareto-Optimum ab, das heißt es gibt kein Verbesserungspotential für beide Akteursgruppen. Die sogenannte Tauschlinse ist deshalb leer.

Die Indifferenzkurve $B B$ der Bürger zeigt im Punkt $E$ an, dass sich die Bürger in Sicherheit wiegen und deshalb kaum bereit sind, auf verfügbares Einkommen für eine weiter verbesserte Beherrschung von Risiken zu opfern. Mögliche Heterogenitäten der Präferenzen werden vernachlässigt; diese Vereinfachung erscheint zulässig, weil im Folgenden alle Einwohner des Landes von der CoronaKrise ähnlich betroffen werden. Auch dann könnte allerdings die in Abbildung 2 gezeigte Verformung der Indifferenzkurve zu $B B^{\prime \prime}$ vom Ausmaß der Risikoaversion abhängen, das sich zwischen den Bürgern unterscheiden dürfte (vgl. jedoch die Bemerkung zu Gleichung (A.5) im Anhang).

- Ein Indikator der Beherrschung von Risiken ist die Luftqualität, gemessen als Konzentration feiner Partikel in $\mu \mathrm{g} / \mathrm{m}^{3}$. Hier sind Deutschland und Frankreich mit 19 bzw. $20 \mu \mathrm{g} / \mathrm{m}^{3}$ im Mittelfeld der EU, allerdings weit hinter Schweden mit $10 \mu \mathrm{g} / \mathrm{m}^{3}$ (European Commission, 2019). Die EU stipuliert $25 \mu \mathrm{g} / \mathrm{m}^{3}$ als zulässigen Grenzwert, die Weltgesundheitsorganisation (WHO) sogar nur $10 \mu \mathrm{g} / \mathrm{m}^{3}$ (European Commission 2019).

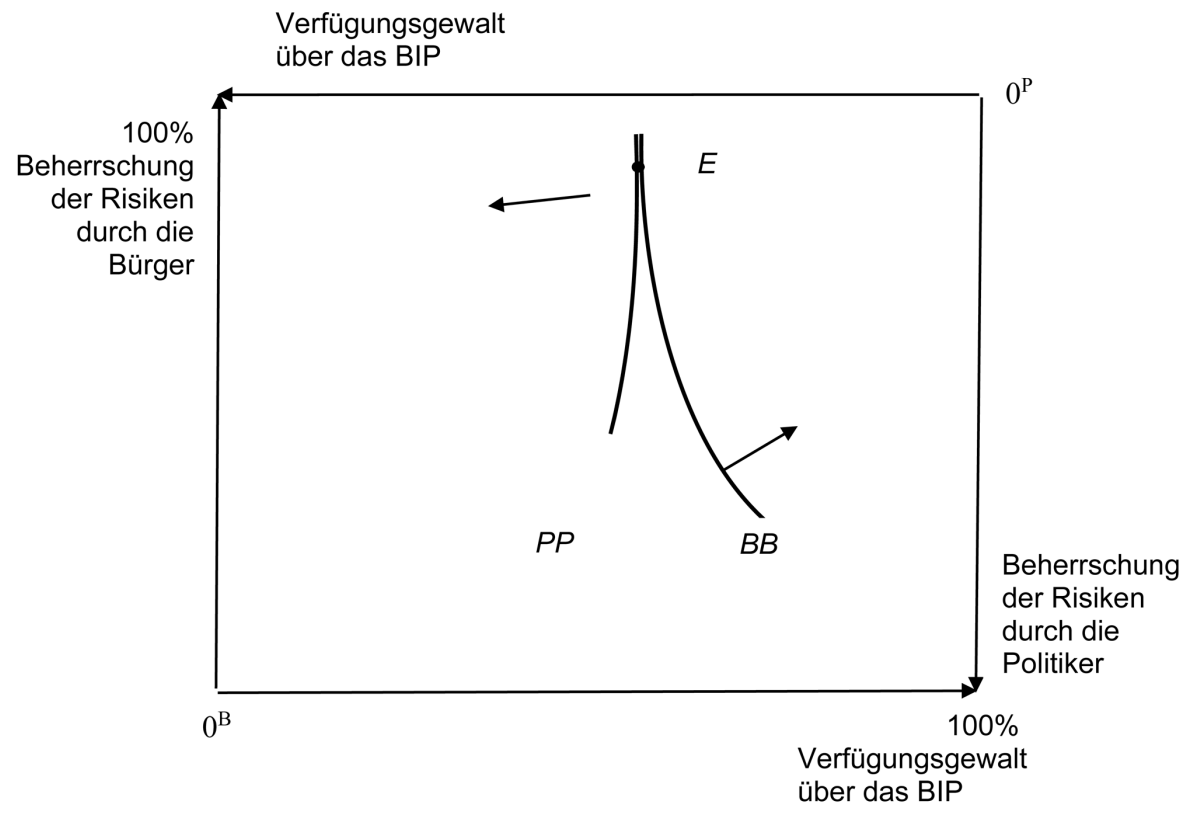

Abbildung 1: Edgeworth-Box mit Präferenzen der Bürger $(B B)$ und der Politiker $(P P)$ vor dem Eintreten von Corona Quelle: Eigene Darstellung 
- Ein anderer Indikator sind die Unfälle im Straßenverkehr: Hier wies Deutschland im Jahr 201638 Tote je Million Einwohner auf, Frankreich 54, Schweden 27 (European Commission 2018). In beiden Fällen bestimmt das individuelle Verhalten (Rauchen, Shopping in der Innenstadt, Fahrverhalten) das Risiko in beträchtlichem Maße, was die vertikale Lage von Punkt $E$ begründet.

- Dies trifft auf die Atomenergie weniger zu, dafür existiert aber eine Schätzung der individuellen Zahlungsbereitschaft für eine Reduktion zumindest des mit ihr verbundenen finanziellen Risikos. Sie wurde für den Fall eines katastrophalen Reaktorunfalls (GAU, Größter Anzunehmender Unfall) mittels eines Discrete Choice Experiments (DCE) erhoben (Schneider und Zweifel, 2013, 2004)1. Für eine Erhöhung der Versicherungsdeckung um einen Prozentpunkt des Schadens betrug die geschätzte marginale Zahlungsbereitschaft nur gerade 5 Dollar pro Jahr bzw. 0,14 Cent pro Kilowattstunde (5,30 Euro bzw. 0,144 Eurocent zu Wechselkursen von 2002). Diese Schätzung bestätigt (zumindest für die Schweiz und vor Fukushima 2011) den praktisch vertikalen Verlauf der Indifferenzkurve $B B$ im Punkt $E$ der Abbildung 1.

Die Indifferenzkurve $P P$ spiegelt die Präferenzen der Politiker wider, wobei mögliche Heterogenitäten zwischen ihnen wiederum ausgeblendet sind. Ihr senkrechter Verlauf in der Umgebung von $E$ lässt erkennen, dass die Politiker bei einer weitgehenden Beherrschung der Risiken kaum bereit sind, auf öffentliche Einnahmen (und damit auf Kompetenzen und Einfluss) zu verzichten. Die horizontale Lage des Ausgangspunkts $E$ lässt sich wiederum durch Indikatoren beschreiben.

- Erstens betrug die deutsche Staatsquote 2019 gemäß der OECD (2020) 45 Prozent des Bruttoinlandsprodukts (in der Schweiz waren es 34 Prozent; das Maximum der Industrieländer erreichte Frankreich mit 56 Prozent, die Vereinigten Staaten standen bei 38 Prozent).

1 Im Gegensatz zur Contingent-Valuation-Methode werden in einem DCE alle Attribute des betrachteten Guts variiert. In der zitierten Studie waren dies neben dem Preis je Kilowattstunde, die Zahl der Stromunterbrechungen je Jahr, Abfallbeseitigung gelöst ja/nein, Schadenhöhe bei einem katastrophalen Unfall sowie die prozentuale Versicherungsdeckung. Die wiederholte Wahl zwischen dem Status quo und einer Alternative mit variierenden Attributen erlaubt die ökonometrische Schätzung der marginalen Zahlungsbereitschaft für die einzelnen Attribute sowie für das Gut insgesamt.
- Zweitens vergibt Freedom House (2018) Punkte für die bürgerlichen Freiheiten, die „Civil Liberties“. Hier befand sich Deutschland 2017 mit 57 von 60 Punkten in der Spitzengruppe, gefolgt von Frankreich mit 52 und knapp hinter der Schweiz mit 57 Punkten (zum Vergleich: Die Vereinigten Staaten erreichten 53 Punkte).

- Drittens publiziert die Weltbank jährlich einen Bericht über die Leichtigkeit, ein Geschäft zu gründen oder weiterzuführen (Weltbank 2020). Auch wenn das verfügbare Einkommen der Bürger einen großen Anteil am BIP ausmacht, heißt das noch nicht, dass sie wirklich frei darüber verfügen können. Von 190 Ländern stand 2019 (also vor Ausbruch der Corona-Krise) Deutschland auf Rang 22, Frankreich auf Rang 32, noch vor der Schweiz im Rang 36 (die Vereinigten Staaten schneiden mit Rang 6 besonders gut ab, dicht gefolgt von Schweden auf Platz 10).

Folgerung 1: Vor dem Ausbrauch der Corona-Krise lässt sich die Aufteilung der Güter „Beherrschung von Risiken“ und „Verfügungsgewalt über das BIP“ durch einen Punkt in der Edgeworth-Box beschreiben, der eine weitgehende Risikobeherrschung durch die Bürger und eine ungefähr hälftige Aufteilung des BIP anzeigt.

\subsection{Die erste Corona-Welle}

Mit dem Corona-Virus trat Anfang 2020 ein neues, zunächst als unbekannt eingestuftes Risiko auf. In der Abbildung 2 ändern sich gegenüber der Abbildung 1 drei Dinge. Zum einen war noch vor dem Eingreifen der Politiker klar, dass das BIP wegen gesunkener Investitions- und Konsumneigung schrumpfen würde; die Edgeworth-Box ist deshalb in der Waagerechten kürzer (zugegebenermaßen wäre diese Verkürzung wohl noch ausgeprägter, wenn die Ausweitung der Staatsausgaben ausgeblieben wäre). Aber auch in Bezug auf eine vergrößerte Grundgesamtheit der möglichen Risiken ist jetzt das Ausmaß an Kontrolle insgesamt geringer; die Box ist deshalb in der Senkrechten ebenfalls kleiner. Drittens ist der Ausgangspunkt $E$ (in Abbildung 2 zusammen mit gestrichelten Indifferenzkurven zum Vergleich eingetragen) nicht mehr zu halten. Der neue Status quo ist jetzt durch $F$ gegeben, mit zunächst unveränderter hälftiger Aufteilung des BIP zwischen Bürgern und Politikern.

Wie im Anhang gezeigt wird, hat die Zunahme des Ausgangsrisikos unter realistischen Voraussetzungen eine Verflachung der Indifferenzkurve $B B$ zur Folge. Der Grund liegt darin, dass die Bürger jetzt bereit sind, im Austausch für verbesserte Beherrschung von Risiken mehr von ihrem 
Verfügungsgewalt

über das BIP

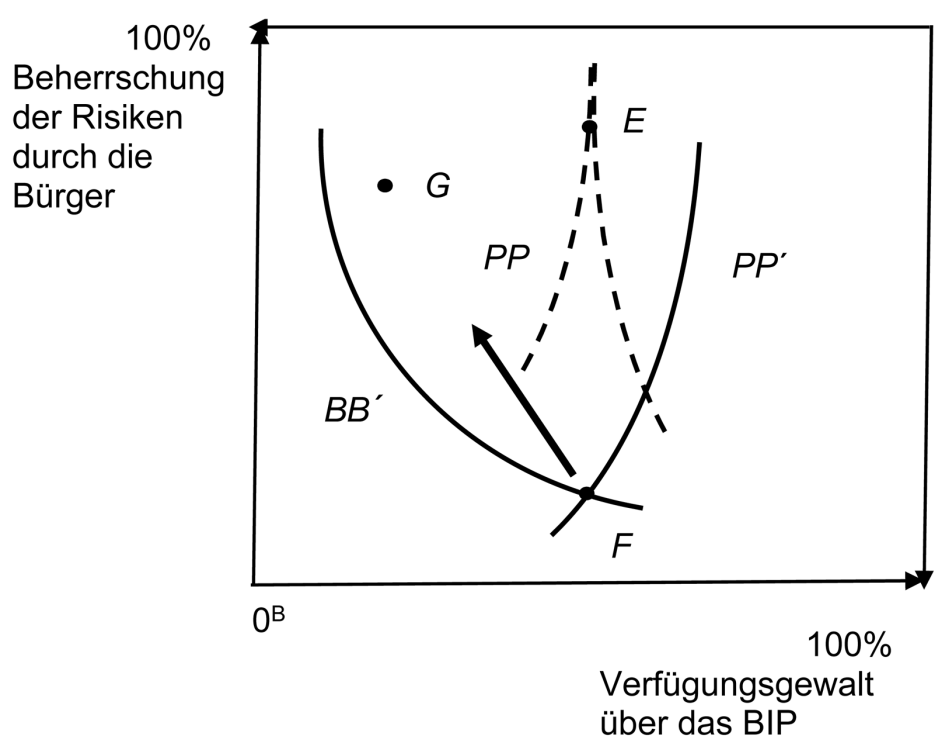

$0^{P}$

Beherrschung

der Risiken

durch die

Politiker

Abbildung 2: Edgeworth- Box mit Präferenzen der Bürger $(B B)$ und der Politiker $(P P)$ nach dem Eintreten der ersten Corona-Welle Quelle: Eigene Darstellung

Einkommen (und damit Kompetenzen und Macht) an die Politiker abzutreten. Die ausgezogene Indifferenzkurve $B B$ in Abbildung 2 verläuft demnach flacher als die ursprüngliche.

Offensichtlich öffnet sich nunmehr eine Tauschlinse. Beide Akteursgruppen können sich besser stellen, wenn die Bürger wieder vermehrt die Kontrolle über die Risiken übernehmen und im Gegenzug einen größeren Anteil am BIP an die Politiker abgeben. Das neue Pareto-Optimum sei zum Beispiel der Punkt $G$; er bildet in der Nach-Corona Zeit den Ausgangspunkt. Der Übergang ist 2020 bereits eingetreten, indem die Regierungen der EU-Länder fast ausnahmslos Hilfeleistungen und Subventionen versprochen haben. Im Falle Deutschlands wurde das öffentliche Budget um 353 Milliarden Euro aufgestockt (Bundesministerium für Finanzen 2020); bei dem zu erwartenden Rückgang des BIP um 9 Prozent (Statista 2020a) wird die Staatsquote von 45,3 Prozent (an einem BIP von 3440 Milliarden Euro 2019) auf rund 61 Prozent steigen [(3440 x 0,453+ $353) /(3440 \times 0,91)=0,612]$.

Folgerung 2: Die Zunahme des Gesundheitsrisikos wegen des Corona-Virus hat eine Zunahme der marginalen Zahlungsbereitschaft der Bürger für eine verbesserte Beherrschung der Risiken zur Folge. Dies wiederum öffnet am neuen Status quo eine Tauschlinse mit Allokationen, die einen größeren Anteil der Politiker am BIP beinhalten.

\subsection{Die „Neue Normalität“" nach der ersten Corona-Welle: Zwei Szenarien}

Dieser Abschnitt enthält eine Voraussage für die Zeit nach Corona. Sie geht aus der Abbildung 3 hervor, wo der neue Ausgangspunkt $G$ aus der Abbildung 2 übernommen wird. Die Dimensionen der Edgeworth-Box sind die gleichen wie in Abbildung 2, was die Annahme widerspiegelt, dass es nach dem Jahr 2020 nicht sofort zu einer Rückkehr des Wachstums kommen wird. Im ersten Fall gehen die Bürger davon aus, dass es keine zweite Corona-Welle geben wird. Dann verlaufen die Indifferenzkurven $B B$ und $P P$ gleich wie in Abbildung 1, da ja die Wahrscheinlichkeit einer Erkrankung wieder auf den ursprünglichen Wert zurückgegangen ist. Das neue Pareto-Optimum ist der Punkt $H$; allerdings wird jetzt schon klar, dass die ursprüngliche Allokation $E$ nicht mehr erreicht werden kann.

In China machte denn auch bereits im März 2020 die Rede von einem „new normal“ die Runde, bei dem die bürgerlichen Freiheiten eingeschränkt bleiben (Kuo 2020). Einmal mehr sind inzwischen Politiker der EU dem chinesischen Vorbild gefolgt. Der italienische Premierminister Conte sagte im Juni: „Wir können zusätzliche Eingriffe nicht ausschließen“ (Antonelli 2020), während Macron in seiner Regierungserklärung eine Rückkehr zum Gaullismus mit seinem starken Staat ins Auge fasste (Chol und Pogam 2020). Aber auch deutsche Politiker haben das Schlagwort übernommen und ebenfalls eine „neue Nor- 


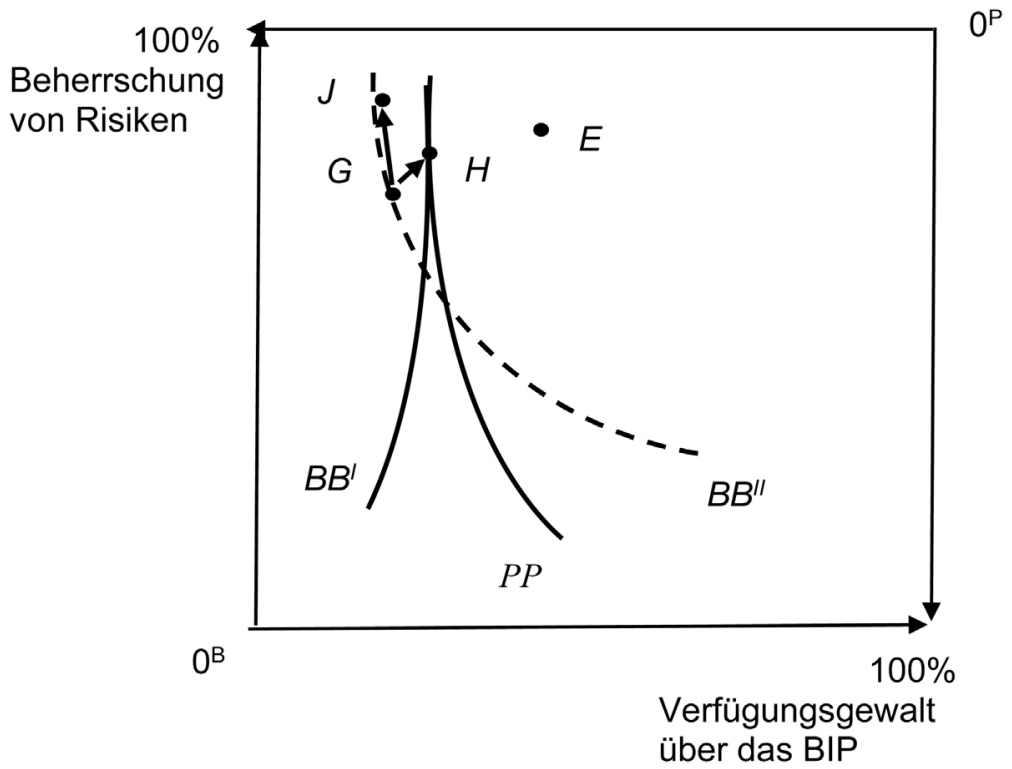

Abbildung 3: Edgeworth-Box mit Präferenzen der Bürger $\left(B B^{\prime}, B B^{\prime \prime}\right)$ und der Politiker $(P P)$ nach dem Ende der ersten Corona-Welle

malität“ ausgerufen (Berger 2020). Sie bedeutet deshalb auch in diesem günstigen ersten Fall, dass die Verfügungsgewalt über das BIP (und damit wohl andere Kompetenzen) vermehrt beim Staat angesiedelt sein wird.

Im zweiten Fall antizipieren die Bürger wie bei der Spanischen Grippe eine zweite Welle, die der Einfachheit halber gleich riskant sein soll wie die erste. Er wird im Anhang ebenfalls analysiert mit dem Ergebnis, dass die marginale Zahlungsbereitschaft der Bürger dadurch nicht notwendig nochmals angehoben wird. Deshalb hat in der Abbildung 3 die Indifferenzkurve $B B^{\prime \prime}$ die gleiche Steigung wie in der Abbildung 2. Bei unveränderten Präferenzen der Politiker öffnet sich beim Punkt $\mathrm{G}$ eine weitere Tauschlinse (nicht eingetragen), mit der Folge, dass es nochmals zu einem vergrößerten Anteil der Politiker an der Verfügung über das BIP kommt. Im Gegenzug erlangen die Bürger eine nochmals erhöhte Kontrolle über die Risiken.

Folgerung 3: Nach dem Abklingen der ersten Corona-Welle sind zwei Szenarien zu unterscheiden. Im einen rechnen die Bürger nicht mit einer Wiederholung, so dass ihre marginale Zahlungsbereitschaft für die Beherrschung von Risiken auf den ursprünglichen Wert zurückgeht. Dennoch kann die ursprüngliche Aufteilung des BIP nicht mehr erreicht werden. Im zweiten Fall antizipieren die Bürger eine zweite Welle, so dass ihre marginale Zahlungsbereitschaft erhöht bleibt. Das Pareto-Optimum entfernt sich dann noch mehr von der ursprünglichen Allokation zu Lasten der Bürger.

\section{Abschließende Bemerkungen}

Das Ziel dieses Beitrags ist es, eine Antwort auf die Frage zu finden, weshalb sonst freiheitsliebende Bürger im Zuge der Corona-Krise ihren Regierungen widerstandslos gestatten, ihre Kompetenzen massiv auszudehnen. Das verwendete Instrument der Analyse ist die Edgeworth-Box mit einer ungefähr hälftigen Aufteilung der Verfügungsgewalt über das BIP zwischen den Bürgern und den Politikern in der Ausgangslage. Die Beherrschung von Risiken wird von beiden Akteursgruppen als praktisch vollständig eingeschätzt (Folgerung 1).

Mit dem Eintreten der ersten Corona-Welle nimmt die marginale Zahlungsbereitschaft der Bürger für eine verbesserte Beherrschung von Risiken zu. Damit öffnet sich am neuen Status quo eine Tauschlinse mit Allokationen, die einen größeren Anteil der Politiker am BIP beinhalten (Folgerung 2). Am Ende der ersten Corona-Welle rechnen die Bürger in einem ersten Szenario nicht mit einer zweiten Welle, so dass ihre marginale Zahlungsbereitschaft für die Beherrschung von Risiken auf den ursprünglichen Wert zurückgeht. Im zweiten Fall antizipieren die Bürger eine zweite Welle, und ihre marginale Zahlungsbereitschaft bleibt erhöht. Das ursprüngliche Pareto-Optimum ist in beiden Fällen nicht mehr erreichbar; es bleibt bei einer ausgeweiteten Kontrolle der Politiker über das BIP im Austausch gegen eine verbesserte Beherrschung der Risiken (Folgerung 3).

Selbstverständlich unterliegt diese Skizze mehreren Einschränkungen. So könnte die erhöhte Bereitschaft der Bürger, verfügbares Einkommen an die Politiker abzuge- 
ben, ein Ausdruck der Solidaritätswelle sein, die nach verschiedenen Medienberichten die EU-Länder erfasste. Dann würden die Politiker im expliziten Auftrag der Bürger und nicht im Eigeninteresse handeln, wenn sie ihre Budgets massiv ausweiten. Ein zweiter denkbarer Kritikpunkt ist die Vernachlässigung der Politikberatung durch Repräsentanten des Gesundheitswesens, allen voran durch Epidemiologen. Ihre so nicht eingetretenen hohen Voraussagen der Todesfälle haben vermutlich zu einer Überschätzung des Rückgangs in der Beherrschung der Risiken geführt ${ }^{2}$. Es ist auch fraglich, ob die eingetragenen Pareto-Optima tatsächlich erreicht werden; der modellhafte Markt, auf dem politischer Einfluss gehandelt werden kann, ist immerhin von beträchtlichen Handelshemmnissen gekennzeichnet und findet am ehesten an den Wahlterminen statt.

Trotz dieser Unvollkommenheiten vermag die politökonomische Betrachtung die Vorgänge im Zusammenhang mit der Corona-Krise zumindest ein Stück weit zu erklären. Sie sagt in Bezug auf die „neue Normalität“ eine bleibende Ausdehnung der Staatsquote und damit wohl auch eine Einschränkung der Menschen in ihrer Ausübung der bürgerlichen Rechte voraus.

Danksagung: Der Autor dankt seiner Frau Katharina Genau Zweifel für ihre sorgfältige Durchsicht des Manuskripts und einem anonymen Gutachter für Kritikpunkte, die zu einer Reihe von Klarstellungen geführt haben.

2 So lautete die ursprüngliche Prognose von Drosten (2020) auf fast 280.000 zusätzliche Todesfälle in Deutschland; bis zum 7. August 2020 gab es nach der Zählung der Johns Hopkins University aber nur 9181 (Coronavirus.jhu.edu/region/Germany). Natürlich kann man argumentieren, es seien die massiven Eingriffe gewesen, die eine so hohe Zahl der Todesfälle verhindert hätten (Präventionsparadoxon). Allerdings ging die Wachstumsrate der Infektionszahlen wohl bereits Mitte März zurück (medscience 2020). Ebenso ist fraglich, ob die besonders zu schützenden älteren Menschen wirklich profitiert haben; eine japanische Studie an über 65-Jährigen (Aida et al. 2011) brachte deren Sterblichkeit mit der Aussage „Ich treffe Freunde selten", einem wichtigen Indikator des Sozialkapitals, in Verbindung. Die Autoren kommen auf einen Anstieg der jährlichen Sterblichkeit von 1,4 auf 1,82 Prozent. Unter der (konservativen) Annahme, dass 10 Prozent der 18,01 Millionen über 65-Jährigen in Deutschland (Statista $2020 b)$ von der Isolation betroffen werden, bedeutet dies $7564(=18,01$ Millionen x 0,1 x 0,0042) zusätzliche Todesfälle. Die Isolationsmaßnahmen laufen damit Gefahr, unter den als besonders schützenswerten älteren Einwohnern mehr Leben dahinzuraffen als zu retten.

\section{Literaturverzeichnis}

Aida, J. et al. (2011), Assessing the association between all-cause mortality and multiple aspects of individual social capital among the older Japanese, BMC Public Health 11, Nr. 499.

Antonelli, L. N. (2020), Stati Generali, Conte: A settembre piano Revovery Italia. Non possiamo escludere altri interventi, Finanza.com, 16. Juni, online verfügbar unter https://www.finanza.com/Finan za/Notizie_Italia/Italia/notizia/Stati_Generali_Conte_a_sette mbre_piano_Recovery_Italia_N-521250.

Barras, V. und G. Greub (2014), History of biological warfare and bioterrorism, Clinical Microbiology and Infection 20(6), S. 497-502.

Berger, David (2020), „Neue Normalität“: Es wird kein Zurück hinter die Abschaffung fundamentaler Grundrechte geben!, online verfügbar unter https://philosophia-perennis.com/2020/04/ 18/neue-normalitaet-covid20/, 5. Juli.

Bundesministerium für Finanzen (2020), Kampf gegen Corona: Größtes Hilfspaket in der Geschichte Deutschlands, online verfügbar unter https://www.bundesfinanzministerium.de/ Content/DE/Standardartikel/Themen/Schlaglichter/CoronaSchutzschild/2020-03-13-Milliarden-Schutzschild-fuerDeutschland.html.

Chol, E. und P. Pogam (2020), Macron, du „nouveau monde“ à la „nouvelle socièté“, L'Express vom 17. Juni, online verfügbar unter https://www.lexpress.fr/actualite/politique/macron-dunouveau-monde-a-la-nouvelle-societe_2128374.html.

Deutsches Ärzteblatt (2019), Grippewelle war tödlichste seit 30 Jahren, 30. September.

Focus (2020), 278.000 Virus-Tote in Deutschland? Wie der Top-Virologe seine Zahl meint, 13. März, online verfügbar unter https:// www.focus.de/gesundheit/news/pandemie-virologe-klaertueber-neuartiges-virus-auf-immunitaet-sterblichkeit-dauer_ id_11723764.html.

European Commission (2018), Road safety: Data show improvement in 2017 but renewed efforts are needed for further substantial progress, Mitteilung vom 10. April, online verfügbar unter https://ec.europa.eu/commission/presscorner/detail/en/ IP_18_2761.

European Commission (2019), Cleaner air for all, Infografik, online verfügbar unter https://ec.europa.eu/environment/air/ cleaner_air/.

Farrell, P. (2020), Spanish Flu: Why did it go away, and might that apply with COVID-19?, online verfügbar unter https://medium. com/@drpatfarrell/spanish-flu-why-did-it-go-away-and-mightthat-apply-with-covid-19-33 f.17b23471.

Finanznachrichten (2020), Deutschland: BIP sinkt im 2. Quartal um $14 \%, 2020$ Rückgang um $9 \%$, online verfügbar unter https:// www.finanznachrichten.de/nachrichten-2020-05/49614867deutschland-bip-sinkt-im-2-quartal-um-14-2020-rueckgang-um9-545.htm.

Finkelstein, A., E. F. P. Luttmer und M. J. Notowidigdo (2013), What good is wealth without health? The effect of health on the marginal utility of consumption, Journal of the European Economic Association 11(1), S. 221-58.

Fischer, B., H. Telser und P. Zweifel (2018), End-of-life healthcare expenditure: Testing economic explanations using a Discrete Choice Experiment, Journal of Health Economics 60, S. 30-38. Gesamtverband der Deutschen Versicherungswirtschaft (2019), Statistisches Taschenbuch der Versicherungswirtschaft 2019, 
online verfügbar unter https://www.gdv.de/resource/blob/ 50214/29985281b05d0ce23240d7b7bdff57cd/downloadstatistisches-taschenbuch-2019-data.pdf.

Kuo, L. (2020), „The new normal“: China's excessive coronavirus public monitoring could be here to stay, The Guardian vom 9. März.

medscience (2020), SARS-Cov2 infection growth rate in Germany, online verfügbar unter https://medscience.one/corona/ germany.

OECD (2020), General government spending (indicator), online verfügbar unter https://data.oecd.org/gga/general-govern ment-spending.htm.

OECD (2018), Health spending, online verfügbar unter https://data. oecd.org/healthres/health-spending.htm.

Schneider, Y. und P. Zweifel (2004), How much internalization of risk through liability insurance?, Journal of Risk and Uncertainty 29, S. $219-40$.

Schneider, Y., P. Zweifel (2013), Spatial effects in willingness to pay for avoiding nuclear risks, Swiss Journal of Economics and Statistics 149(3), S. 357-79.

Statista (2020a), Statistiken zum Bruttoinlandsprodukt (BIP) und Wirtschaftswachstum, online verfügbar unter https://de. statista.com/themen/26/bip/,17. März.

Statista (2020b), Bevölkerung - Zahl der Einwohner in Deutschland nach relevanten Altersgruppen am 31. Dezember 2019, online verfügbar unter https://de.statista.com/statistik/daten/studie/ 1365/umfrage/bevoelkerung-deutschlands-nach-altersgruppen $/, 10$. August.

Tribelhorn, M. (2020), Vor 100 Jahren wütete die Spanische Grippe. Bis heute bleibt sie ein Rätsel, Neue Zürcher Zeitung vom 16. März, online verfügbar unter https://www.nzz.ch/gesellschaft/spanische-grippe-1918-ein-toedliches-fieber-geht-umdie-welt-ld.1366421?reduced=true.

Tuttitalia (2019), Cinesi in provincia di BG, online verfügbar unter https://www.tuttitalia.it/lombardia/provincia-di-bergamo/ statistiche/cittadini-stranieri/repubblica-popolare-cinese/, 20. Juli.

Weltbank (2020), Doing Business 2020, online verfügbar unter https://openknowledge. worldbank.org/bitstream/handle/ 10986/32436/9781464814402.pdf.

\section{Anhang}

In diesem Anhang wird gezeigt, dass ein erhöhtes Gesundheitsrisiko in der Ausgangsperiode auf Seiten der Bürger eine Zunahme der marginalen Zahlungsbereitschaft für die Beherrschung von Risiken bewirkt. Um die zweite Welle analog zur Spanischen Grippe abzubilden, ist der Erwartungsnutzen $E U$ über zwei Perioden von rund sechs Monaten entsprechend dem Infektionszyklus definiert. Additiv und einfachheitshalber ohne Diskontierung ist er gegeben durch

$E U^{B}=\pi_{1} \cdot v^{R}\left[Y_{1}\right]+\left(1-\pi_{1}\right) v^{N R}\left[Y_{1}\right]+\pi_{2} \cdot v^{R}\left[Y_{2}\right]+\left(1-\pi_{2}\right) v^{N R}\left[Y_{2}\right]$, (A.1) mit $\pi_{1}$ : Wahrscheinlichkeit eines Schadens in der laufenden $\left(\pi_{2}\right.$ : in der zweiten) Periode, $Y_{1}$ : Einkommen in der laufenden $\left(\mathrm{Y}_{2}\right.$ : zweiten) Periode und $v^{\mathrm{R}}($.): die zustandsabhängige Risiko-(von-Neumann-Morgenstern-)Nutzenfunktion bei Eintreten [ $v^{\mathrm{NR}}($.$) : ohne Eintreten] des Risikos.$ Zur Herleitung des Trade-offs zwischen einem Opfer an Einkommen und der Senkung der verbesserten Beherrschung des Risikos (ausgedrückt als Senkung der Wahrscheinlichkeit, ihm ausgesetzt zu sein), sind zwei Varianten zu unterscheiden: Zum einen soll die Veränderung des Risikos nur die laufende Periode betreffen $\left(d \pi_{1}>0, d \pi_{2}=0\right.$, das heißt eine zweite Welle wird nicht erwartet); zum anderen soll sie in beiden Perioden gleich groß sein, also $\mathrm{d} \pi_{1}=\mathrm{d} \pi_{2}:=\mathrm{d} \pi$. Unabhängig davon schrumpft das Einkommen, so dass in der Abbildung 3 die Edgeworth-Box um den Betrag dYverkürzt ist, so dass $d Y_{1}=d Y_{2}:=d Y<0$. Die Gleichung der ursprünglichen Indifferenzkurve $B B$ in $A b$ bildung 1 (gestrichelt in Abbildung 2) ist im ersten Fall gegeben durch

$d E U^{B}=0=d \pi \cdot v^{R}\left[Y_{1}\right]+\pi_{1} \cdot \frac{\delta v^{R}}{\delta Y}\left[Y_{1}\right] d Y-d \pi_{1} \cdot v^{N R}\left[Y_{1}\right]-\pi_{1} \cdot \frac{\delta v^{N R}}{\delta Y}\left[Y_{1}\right] d Y_{1}$

wobei zu beachten ist, dass sowohl der Nutzen wie auch der Grenznutzen an den jeweiligen Werten des Vermögens gemessen werden müssen. Die Steigung der Indifferenzkurve in der Ausgangslage beträgt somit

$\left[\frac{d \pi_{1}}{d Y_{1}}\right]^{B, I}=-\frac{\pi_{1} \frac{\delta u^{R}}{\delta Y}\left[Y_{1}\right]-\pi_{1} \frac{\delta v^{N R}}{\delta Y}\left[Y_{1}\right]}{v^{R}\left[Y_{1}\right]-v^{N R}\left[Y_{1}\right]}==\frac{\pi_{1}\left\{\frac{\delta v^{N R}}{\delta Y}\left[Y_{1}\right]-\frac{\delta v^{R}}{\delta Y}\left[Y_{1}\right]\right\}}{\left\{v^{R}\left[Y_{1}\right]-v^{N R}\left[Y_{1}\right]\right\}}$

bzw.

$\left[\frac{d \pi\left(1-\pi_{1}\right)}{d Y_{1}}\right]^{B, I}=-\frac{\pi_{1}\left\{\frac{\delta u^{N R}}{\delta W}\left[Y_{1}\right]-\frac{\delta v^{R}}{\delta Y}\left[Y_{1}\right]\right\}}{\left\{v^{R}\left[Y_{1}\right]-v^{N R}\left[Y_{1}\right]\right\}}<0$.

Das negative Vorzeichen gilt unter den folgenden Voraussetzungen. Der Nenner der Gleichung (A.3) ist negativ, denn bei einem gegebenen Vermögen übersteigt der Nutzen bei Nichteintreten des Risikos als jenen bei seinem Eintreten. Das Vorzeichen des Zählers (vor Multiplikation mit -1) hängt davon ab, wie der Grenznutzen des Einkommens mit dem Eintreten des Risikos variiert. Vielfach gilt auf Grund der Schätzungen von Finkelstein et al. (2009) der Grenznutzen bei Nichteintreten des Risikos als der höhere, so dass $\delta v^{N R} / \delta Y\left[Y_{1}\right] />\delta v^{R} / \delta Y\left[Y_{1}\right]$.. Dann hätte die Indifferenzkurve $B B$ allerdings positive Steigung, obschon sie wie üblich über zwei Güter definiert ist. Wenn jedoch die Nutzendifferenz zwischen $v^{N R}\left(\right.$.) und $v^{R}($.) dann hoch ist, wenn sowohl das Einkommen wie auch die Gesundheit 
unter dem Erwartungswert sind, muss $\delta v^{N R} / \delta Y\left[Y_{1}\right] /<\delta v^{R} /$ $\delta Y\left[Y_{1}\right]$ sein (Eeckhoudt und Schlesinger 2006) ${ }^{3}$. Nur so kann sich die niedriger gelegene Funktion $v^{R}($.) von unten an $v^{N R}($.) annähern, wenn das Einkommen zunimmt. Damit hat der Zähler in Gleichung (A.3) ein positives Vorzeichen und die Indifferenzkurve $B B$ negative Steigung. Sie zeigt an, dass die Bürger für eine Verbesserung der Risikobeherrschung bereit sind, auf ihren Anteil am BIP etwas zu verzichten.

Wegen des Corona-Virus hat jedoch das Gesundheitsrisiko zugenommen. Dies hat den folgenden Effekt auf die Steigung der Indifferenzkurve:

$$
\frac{d}{d \pi_{1}}\left[\frac{d\left(1-\pi_{1}\right)}{d Y_{1}}\right]^{B, I}=-\frac{\left\{\frac{\delta v^{N R}}{\delta Y}\left[Y_{1}\right]-\frac{\delta v^{R}}{\delta Y}\left[Y_{1}\right]\right\}}{\left\{v^{R}\left[Y_{1}\right]-v^{N R}\left[Y_{1}\right]\right\}}>0,
$$

da Nenner und Zähler (vor Multiplikation mit 1) wiederum negativ sind. In der Abbildung 2 verläuft damit die Indifferenzkurve $B B^{\prime}$ flacher als in der Abbildung 1. Das Ausmaß der Verflachung könnte von der Risikoaversion der Bürger abhängen. Dann müsste aber in Gleichung (A.5) der Zähler mit der Risikoaversion (also $\delta^{2} v^{R} / \delta Y^{2}<0$ bzw. $\delta^{2} v^{N R} / \delta Y^{2}<0$ ) zunehmen und/oder der Nenner abnehmen. Dies ist jedoch nicht der Fall, weil $\delta v^{R} / \delta Y, \delta v^{N R} / \delta Y$, und $v^{R}$ sowie $v^{N R}$ alle an der festen Stelle $Y_{1}$ auszuwerten sind. Die zweite Ableitung kommt erst bei der Kurvatur der Indifferenzkurve zum Tragen, die jedoch für die Argumentation nicht entscheidend ist.

Im zweiten Fall (eine zweite Welle wird erwartet) erhält man mit $d \pi_{1}=d \pi_{2}:=d \pi$

$$
\begin{aligned}
& d E U^{B}=0=d \pi \cdot v^{R}\left[Y_{1}\right]+\pi_{1} \cdot \frac{\delta v^{R}}{\delta Y}\left[Y_{1}\right] d Y-d \pi \cdot v^{N R}\left[Y_{1}\right]-\pi_{1} \cdot \frac{\delta v^{N R}}{\delta Y}\left[Y_{1}\right] d Y \\
& +d \pi \cdot v^{R}\left[Y_{2}\right]+\pi_{2} \cdot \frac{\delta v^{R}}{\delta Y}\left[Y_{2}\right] d Y-d \pi \cdot v^{N R}\left[Y_{2}\right]-\pi_{2} \cdot \frac{\delta v^{N R}}{\delta Y}\left[Y_{2}\right] d Y, \quad \text { (A.6) }
\end{aligned}
$$

und damit für die Steigung der Indifferenzkurve $B B$

$$
\begin{gathered}
{\left[\frac{d \pi}{d Y}\right]^{B, I}=-\frac{\pi_{1} \frac{\delta v^{R}}{\delta Y}\left[Y_{1}\right]-\pi_{1} \frac{\delta v^{N R}}{\delta Y}\left[Y_{1}\right]+\pi_{2} \frac{\delta v^{R}}{\delta Y}\left[Y_{2}\right]-\pi_{2} \frac{\delta v^{N R}}{\delta Y}\left[Y_{2}\right]}{v^{R}\left[Y_{1}\right]-v^{N R}\left[Y_{1}\right]+v^{R}\left[Y_{2}\right]-v^{N R}\left[Y_{2}\right]}=} \\
=\frac{\pi_{1}\left\{\frac{\delta v^{N R}}{\delta Y}\left[Y_{1}\right]-\frac{\delta v^{R}}{\delta Y}\left[Y_{1}\right]\right\}+\pi_{2}\left\{\frac{\delta v^{N R}}{\delta Y}\left[Y_{2}\right]-\frac{\delta v^{R}}{\delta Y}\left[Y_{2}\right]\right\}}{\left\{v^{R}\left[Y_{1}\right]-v^{N R}\left[Y_{1}\right]\right\}+\left\{v^{R}\left[Y_{2}\right]-v^{N R}\left[Y_{2}\right]\right\}} \text { bzw. } \\
{\left[\frac{d \pi(1-\pi)}{d Y}\right]^{B, I I}=-\frac{\pi_{1}\left\{\frac{\delta v^{N R}}{\delta W}\left[Y_{1}\right]-\frac{\delta v^{R}}{\delta Y}\left[Y_{1}\right]\right\}+\pi_{2}\left\{\frac{\delta v^{N R}}{\delta Y}\left[Y_{2}\right]-\frac{\delta v^{R}}{\delta W}\left[Y_{2}\right]\right\}}{\left\{v^{R}\left[Y_{1}\right]-v^{N R}\left[Y_{1}\right]\right\}+\left\{v^{R}\left[Y_{2}\right]-v^{N R}\left[Y_{2}\right]\right\}}<0 ;}
\end{gathered}
$$

die negative Steigung folgt aus den gleichen Überlegungen wie im ersten Fall.

Weil jetzt die Risikoerhöhung als nachhaltig eingeschätzt wird, gilt $d \pi_{1}=d \pi_{2}:=d \pi>0$ :

$$
\frac{d}{d \pi}\left[\frac{d(1-\pi)}{d Y}\right]^{B, I I}=-\frac{\left\{\frac{\delta v^{N R}}{\delta Y}\left[Y_{1}\right]-\frac{\delta U^{R}}{\delta Y}\left[Y_{1}\right]\right\}+\left\{\frac{\delta v^{N R}}{\delta Y}\left[Y_{2}\right]-\frac{\delta v^{R}}{\delta W}\left[Y_{2}\right]\right\}}{\left\{v^{R}\left[Y_{1}\right]-v^{N R}\left[Y_{1}\right]\right\}+\left\{v^{R}\left[Y_{2}\right]-v^{N R}\left[Y_{2}\right]\right\}}>0
$$

analog zur Gleichung (A.3). Man könnte nun erwarten, dass die Verflachung der Indifferenzkurve $B B$ in Abbildung 2 noch ausgeprägter ist, weil die Erhöhung des Risikos nachhaltiger wirkt. Doch dies ist nicht eindeutig der Fall, denn sowohl Nenner und Zähler nehmen im Absolutwert zu. Die intuitive Erwartung wird dann bestätigt, wenn der zusätzliche Summand im Zähler hoch ist im Vergleich zum Nenner ${ }^{4}$. Dies bedingt dass $v^{R}\left[Y_{2}\right]-v^{N R}\left[Y_{2}\right] \approx 0$, das heißt der Nutzenunterschied zwischen den beiden Zuständen ist gering, während (wieder nach Eeckhoudt und Schlesinger 2006) $\delta v^{N R} / \delta Y\left[Y_{2}\right]-\delta v^{R} / \delta Y\left[Y_{2}\right]$ ? 0, das heißt, der Unterschied im Grenznutzen des Einkommens ist groß. Auf den ersten Blick erscheint dies eine unwahrscheinliche Konstellation, doch ist zu bedenken, dass die Einschränkungen der Konsummöglichkeiten während einer zweiten Welle den Grenznutzen des Einkommens massiv senken könnten. Wegen der mangelnden Eindeutigkeit ist jedoch in der Abbildung 3 die gestrichelte Indifferenzkurve $B B^{\prime \prime}$ gleich eingetragen wie in der Abbildung 2.

4 Dies ist eine ungefähre Abschätzung; eine genaue Herleitung ist kompliziert und lohnt den Aufwand nicht.
3 Bei genauer Betrachtung der Studie von Finkelstein et al. (2009) stellt sich heraus, dass die Autoren nur die Gesundheit, nicht aber das Einkommen als risikobehaftet auffassen. Im vorliegenden Zusammenhang sind jedoch beide Aktiva dem Corona-Risiko ausgesetzt. Für weitere Einzelheiten vgl. Fischer et al. 2018. 